\title{
An appropriate Methodand Analysis forDetermination of Energy losses in Compressors
}

\author{
I. Garba \\ Department of Mechanical Engineering Bayero University, Kano
}

\begin{abstract}
This work investigates the energy losses in compressors used inUnited Nigerian Textile Limited, Kaduna. Simulation was carried out using Hysys simulation software in order to validate the experimental results and simulated results obtained. From the Experiment carried out, it was discovered that the power wasted was $2.563 \mathrm{~kW}$. The annual energy and cost savings were found to be $11,700 \mathrm{kWh} / \mathrm{yr}$ and $\$ 99450 / \mathrm{yr}$ respectively. The exit Temperature and Pressure were recorded as $172.8^{\circ} \mathrm{C}$ and $412.0 \mathrm{kPa}$. The results are very helpful for validating the performance of any system.
\end{abstract}

Significances of the Paper: Evaluation of energy losses is very vital in determining the economic analysis of energy systems.

Keywords: Energy savings, Simulation, Compressor, Isentropic work and Polytrophic compression.

\subsection{Basic Calculations}

\section{Introduction}

For a compression process, the system pressure $\mathrm{P}$ is related to the volume $\mathrm{V}$ by:

$P V^{n}=$ Cons $\tan t$

Where:

$\mathrm{n}=$ Exponent

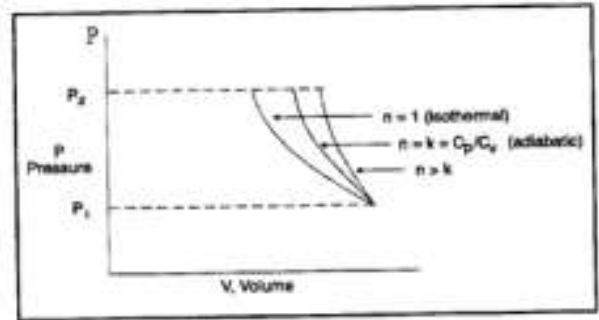

Fig.1 polytropic compression curve

The curve denoted by $n=1$ is an isothermal compression curve. For an ideal gas undergoing adiabatic compression, $\mathrm{n}$ is the ratio of specific heat at constant pressure to that at constant volume, i.e.,

$n=K=C_{P} / C_{v}$

Where:

$k=\quad$ Ideal gas isentropic coefficient

$c_{p}=$ Specific heat at constant pressure

$\mathrm{c}_{\mathrm{v}}=\quad$ Specific heat at constant volume

For a real gas, $\mathrm{n}>\mathrm{k}$.

The Mollier chart (Figure 2) plots the pressure versus the enthalpy, as a function of entropy and temperature. This chart is used to show the methods used to calculate the outlet conditions for the compressor as follows:

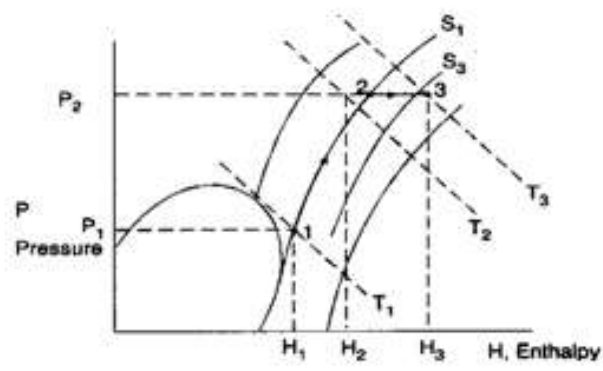

Fig.2:pressure versus the enthalpy 


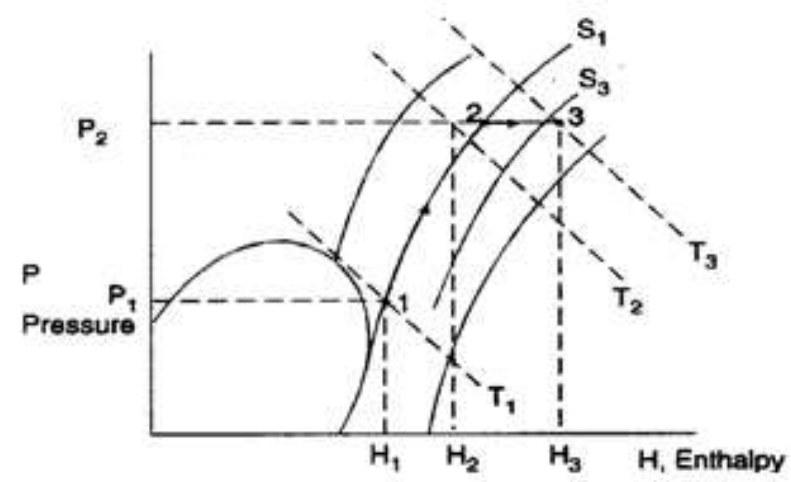

Figure 3 Typical Mollier chart for compression

A flash is performed on the inlet feed at pressure $\mathrm{P}_{1}$, and temperature $\mathrm{T}_{1}$, using a suitable $\mathrm{K}$-value and enthalpy method. The entropy $S_{1}$, and enthalpy $H_{1}$ are obtained and the point $\left(\mathrm{P}_{1}, \mathrm{~T}_{1}, \mathrm{~S}_{1}, \mathrm{H}_{1}\right)$ are obtained.

The constant entropy line through $S_{1}$ is followed until the user-specified outlet pressure is reached. This point represents the temperature $\left(\mathrm{T}_{2}\right)$ and enthalpy conditions $\left(\mathrm{H}_{2}\right)$ for an adiabatic efficiency of $100 \%$. The adiabatic enthalpy change $\Delta \mathrm{H}_{\mathrm{ad}}$ is given by:

$\Delta H_{a d}=H_{2}-H_{1}$

- If the adiabatic efficiency, $\gamma_{a d}$, is given as a value less than $100 \%$, the actual enthalpy change is calculated from:

$$
\Delta H_{a c}=\Delta \frac{H_{a d}}{\gamma_{a d}}
$$

- The actual outlet stream enthalpy is then calculated using:

$$
H_{3}=H_{1}+\Delta H_{a c} \mathrm{H}_{3}
$$

- Point 3 on the Mollier chart, representing the outlet conditions is then obtained. The phase split of the outlet stream is obtained by performing an equilibrium flash at the outlet conditions.

- The isentropic work $\left(\mathrm{W}_{\mathrm{s}}\right)$ performed by the compressor is computed from:

$$
W_{s}=\left(H_{3}-H_{1}\right) \times J=\Delta H_{a c} \times J
$$

Where:

$$
J=\text { mechanical equivalent of energy }
$$

The isentropic power required is:

$$
\begin{aligned}
& G H P_{a d}=\Delta H_{a d} \times 778 \times \frac{F}{33000} \\
& G H P_{a c}=\Delta H_{a c} \times 778 \times \frac{F}{33000}=\frac{G H P_{a d}}{\gamma_{a d}}
\end{aligned}
$$

$H E A D_{a d}=\Delta H \times 778$

Where:

$G H P=$ work, hP

$\Delta \mathrm{H}=$ enthalpy change,

$F=$ mass flow rate,

$H E A D_{a d}=$ Adiabatic Head,

The factor 33000 is used to convert the units into horsepower.

The isentropic and polytropic coefficients, polytropic efficiency, and poly tropic work can be calculated using one of the two methods; the method from the GPSA Engineering Data Book, and the method from the ASME Power Test Code 10.

\section{ASME Method}

This method is more rigorous than the default GPSA method, and yields better results over a wider range of compression ratios and feed compositions.

For a real gas, as previously noted, the isentropic volume exponent (also known as the isentropic coefficient), $\mathrm{n}_{\mathrm{s}}$, is not the same as the compressibility ratio, $\mathrm{k}$. The ASME method distinguishes between $\mathrm{k}$ and $\mathrm{n}_{\mathrm{s}}$ for a real gas. 


\section{Adiabatic Efficiency Given}

In this method, the isentropic coefficient $\mathrm{n}_{\mathrm{s}}$ is calculated as:

$n_{s}=\ln \left(P_{2} / P_{1}\right) / \ln \left(V_{1} / V_{2}\right)$

Where:

$V_{1}=\quad$ Volume at the inlet conditions

$V_{2}=\quad$ Volume at the outlet pressure and inlet entropy conditions

$\mathrm{P}_{1}=\quad$ Pressure at the inlet conditions

$\mathrm{P}_{2}=\quad$ Pressure at the outlet conditions

The compressor work for a real gas is calculated from equation (2.8), and the factor from the following relationship:

$W_{a c}=144\left[n_{s} /\left(n_{s}-1\right)\right] \times f \times P_{1} \times V_{1} \times\left[\left(P_{2} / P_{1}\right)^{\left(n_{s}-1\right) / n_{s}}-1\right]$

The ASME factor $\mathrm{f}$ is usually close to 1 . For a perfect gas, $\mathrm{f}$ is exactly equal to 1 , and the isentropic coefficient $\mathrm{n}_{\mathrm{s}}$ is equal to the compressibility factor $\mathrm{k}$.

The polytropic coefficient, $\mathrm{n}$, is defined by:

$n=\ln \left(P_{2} / P_{1}\right) / \ln \left(V_{1} / V_{2}\right)$

The polytropic work, i.e., the reversible work required to compress the gas in a polytropic compression process from die inlet conditions to the discharge conditions is computed using:

$W_{p}=144\left(\frac{n}{n-1)} \times f \times P_{1} V_{1}\left\{\left(P_{2}-P_{1}\right) \times \frac{n-1}{n}-1\right\}\right.$

Where:

$W_{p}=$ polytropic work

For ideal or perfect gases, the factor $\mathrm{f}$ is equal to 1 . The polytropic efficiency is then calculated by:

$\gamma_{p}=\frac{W_{s}}{W_{p}} \gamma_{\mathrm{p}}$

Where $\mathrm{W}_{\mathrm{s}}=$ isentropic work

This polytropic efficiency will not agree with the value calculated using the GPSA method which is computed using $\gamma_{p}=\{(\mathrm{n}-\mathrm{l}) / \mathrm{n}) /\{(\mathrm{k}-\mathrm{l}) / \mathrm{k}\}$.

\section{GPSA Method}

This GPSA method is the default method, and is more commonly used in the chemical process industry [9].

In this method, the adiabatic head is calculated from equations above. Once this is calculated, the isentropic coefficient $\mathrm{k}$ is computed by trial and error using:

$$
H E A D_{a c}=\left\{\left(Z_{1}+Z_{2}\right) / R T_{1} /\{(k-1)\}\left\{\frac{P_{2}}{P_{1}}\right)^{9 k-1) / k}-1\right\}[39
$$

Where:

$Z_{1}, Z_{2}=$ compressibility factors at the inlet and outlet conditions

$R=$ gas constant

$\mathrm{T}_{1}=\quad$ temperature at inlet conditions.

This trial and error method of computing $\mathrm{k}$ produces inaccurate results when the compression ratio, becomes low. If the calculated compression ratio is less than a value set by the user, the value of $\mathrm{k}$ has to be calculated.If $\mathrm{k}$ does not satisfy $1.0 \leq \mathrm{k} \leq 1.66667$, the isentropic coefficient, is calculated by trial and error based on the following:

$T_{2}=\left(Z_{1} / Z_{2}\right) \times T_{1} \times\left[\left(P_{2} / P_{1}\right)^{(k-1) / k}\right.$

The polytrophic compressor equation is given by:

$$
\left.H E A D_{p}=\left[\left(Z_{1}+Z_{2}\right) / 2\right] \times R T_{1} /\{(n-1) / n\}\right]\left\{\left(P_{2} / P_{1}\right)^{(n-1) / n}-1\right\}
$$

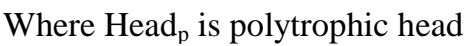

The adiabatic head is related to the polytropic head by:

$$
\frac{H E A D_{a d}}{\gamma_{a d}}=\frac{H E A D_{p}}{\gamma_{p}}
$$

The polytrophic efficiency $\mathrm{n}$ is calculated by: 
$\gamma_{p}=[n /(n-1)] /[k /(k-1)] \gamma_{p}$

The polytropic coefficient, $\mathrm{n}$, the polytropic efficiency $\gamma_{p}$, and the polytropic head are determined by trial and error method. The polytropic gas horsepower is then given by:

$\left.G H P_{p}=H E A D_{p} \times \frac{F}{33000}\right]$

\section{Material And Methods}

The entropy data needed for these calculations are obtained from a number of entropy calculation methods. These include die Soave-Redlich-Kwong cubic equation of state, and the Curl-Pitzer correlation method. Thermodynamic systems may be used to generate entropy data. User-added subroutines may also be used to generate entropy data.Once the entropy data are generated, the condition of the outlet conditions from the compressor and the compressor power requirements are computed, using either a user-input adiabatic or polytrophic efficiency.

III. Results And Discussion

Table 1: Air Facility Reading for 75KI01

\begin{tabular}{|c|c|c|c|c|c|c|c|}
\hline Time & $\begin{array}{l}\text { Air Inlet } \\
\text { Temp. }\left({ }^{0} \mathrm{c}\right)\end{array}$ & $\begin{array}{ll}\text { Oil } & \text { Cooler } \\
\text { L/D } & \text { Temp } \\
\left({ }^{0} \text { c) }\right. & \\
\end{array}$ & $\begin{array}{l}\text { Oil Cooler } \\
\text { L/D } \\
\text { Outlet }\left({ }^{0} \mathrm{c}\right)\end{array}$ & $\begin{array}{lr}\text { After } & \text { Cool } \\
\text { Air } & \text { Outlet } \\
\text { Temp. }\left({ }^{0} \mathrm{c}\right) & \\
\end{array}$ & $\begin{array}{l}\text { Turbine } \\
\text { Inlet Steam } \\
\text { Temp. }\left({ }^{0} \mathrm{c}\right)\end{array}$ & $\begin{array}{l}\text { Turbine Inlet } \\
\text { Steam Pressure } \\
\left(\mathbf{N} / \mathbf{M}^{2}\right)\end{array}$ & $\begin{array}{l}\text { Turbine Exhaust } \\
\text { Pressure }\left(\mathbf{N} / \mathbf{M}^{2}\right)\end{array}$ \\
\hline $8: 00 \mathrm{Hr}$ & 32 & 52 & 30 & 34 & 400 & 41 & 17.1 \\
\hline $10: 00 \mathrm{Hr}$ & 32 & 53 & 31 & 34 & 400 & 42 & 17.3 \\
\hline $11: 00 \mathrm{Hr}$ & 30 & 53 & 31 & 34 & 400 & 42 & 16.9 \\
\hline $12: 00 \mathrm{Hr}$ & 33 & 52 & 32 & 34 & 400 & 42 & 17.0 \\
\hline $14: 00 \mathrm{Hr}$ & 33 & 51 & 32 & 34 & 400 & 41 & 17.3 \\
\hline $15: 00 \mathrm{Hr}$ & 33 & 51 & 30 & 34 & 400 & 41 & 17.2 \\
\hline $16: 00 \mathrm{Hr}$ & 33 & 52 & 32 & 34 & 400 & 42 & 17.2 \\
\hline 17:00 Hr & 33 & 52 & 31 & 34 & 400 & 42 & 17.3 \\
\hline $18: 00 \mathrm{Hr}$ & 33 & 52 & 32 & 34 & 400 & 42 & 17.3 \\
\hline Average & 32.4 & 51.9 & 31.4 & 34 & 400 & 41.5 & $\mathbf{1 7 . 2}$ \\
\hline
\end{tabular}

\section{Compressor Heat Loss}

Assumption: air is an ideal gas. Steady operating condition exists. There is pressure losses:

$$
\begin{gathered}
W_{\text {comp }, \text { in }}=\frac{n R T_{1}}{\eta_{\text {comp }}(n-1)} \times\left(\left(\frac{P_{2}}{P_{1}}\right)^{\frac{(n-1)}{n}}-1\right) \\
W_{\text {comp } \text { in }}=\frac{1.4 \times(0.287) \times 293}{0.8(1.4-1)} \times\left(\left(\frac{801}{101}\right)^{\frac{(0.4)}{1.4}}-1\right)=296.9 K J / K g \\
\begin{array}{l}
\mathrm{A}=\pi \mathrm{D}^{2} / 4=\pi\left(3 \times 10^{-3} \mathrm{~m}^{2}\right) / 4 \\
\text { Air leaking through the hole is determined to be }
\end{array} \\
m_{\text {air }}=C_{\text {discharge }}\left(\frac{2}{K+1}\right)^{\frac{1}{(k-1)}} \times\left(\frac{P_{\text {line }}}{R T_{\text {line }}}\right) A \times \sqrt{K R\left(\frac{2}{K+1}\right) T_{\text {line }}} \\
m_{\text {air }}=0.65\left(\frac{2}{1.4+1}\right)^{\frac{1}{(1.4-1)}} \times\left(\frac{801}{0.287 \times 297}\right) 7.069 \times 10^{-6} \times \sqrt{1.4 \times 0.287\left(\frac{2}{1.4+1}\right) 297} \\
=0.008632 \mathrm{~kg} / \mathrm{s}
\end{gathered}
$$


Then the power wasted by the leaking compressed air becomes

$$
\begin{array}{rlrl}
\text { Power wasted } & =m_{\text {air }} \times W_{\text {comp }, \text { in }} \\
& = & & 0.008632 \times 296.9 \\
& = & & 2.563 \mathrm{~kW}
\end{array}
$$

The compressor operates for 4200 hours a year, and the motor efficiency is 0.92 .

Then the annual energy and cost savings are:

Energy savings $=$ power saved $\mathrm{x}$ operating hours

$$
\begin{aligned}
& \eta_{\text {motor }} \\
& =\underline{2.563 \mathrm{~kW} \mathrm{x} 4200} \\
& 0.92 \\
& =11,700 \mathrm{kWh} / \mathrm{yr}
\end{aligned}
$$

Cost savings $=($ energy savings $) \times($ unit cost of energy $)$

$$
\begin{aligned}
& =11700 \mathrm{x} \$ 8.50 \mathrm{k} / \mathrm{wh} \\
& =\$ 99450 / \mathrm{yr}
\end{aligned}
$$

\section{Compressor Simulation Results:}

Table 2: compressor simulation

\begin{tabular}{|l|l|l|}
\hline S/No & Simulated Parameters & Simulated Values \\
\hline 1. & Duty $(\mathrm{kW})$ & $5.0684 \mathrm{e}+02$ \\
\hline 2. & Polytropic Exponential & 1.626 \\
\hline 3. & Adiabatic Efficiency & 71.72 \\
\hline 4. & Adiabatic Head $(\mathrm{m})$ & 7586 \\
\hline 5. & Isentropic Exponential & 1.402 \\
\hline 6. & Polytropic Efficiency & 74.26 \\
\hline 7. & Polytropic Head (m) & 7852 \\
\hline 8. & Polytropic Head Factor & 1.000 \\
\hline
\end{tabular}

Table 3: Compressor Rating Curves

\begin{tabular}{|l|l|l|l|}
\hline S/NO. & Flow $\left(\mathbf{A C T ~}^{\mathbf{3}} / \mathbf{h}\right)$ & Head $(\mathbf{m})$ & Efficiency $\mathbf{( \% )}$ \\
\hline 1. & 7812 & 7680 & 69.20 \\
\hline 2. & 8388 & 7575 & 72.00 \\
\hline 3. & 8964 & 7841 & 72.48 \\
\hline 4. & 9504 & 7347 & 72.58 \\
\hline 5. & $1.008 \mathrm{e}+004$ & 7153 & 73.08 \\
\hline 6. & $1.062 \mathrm{e}+004$ & 6717 & 72.46 \\
\hline 7. & $1.120 \mathrm{e}+004$ & 5858 & 69.39 \\
\hline 8. & $1.148 \mathrm{e}+004$ & 4957 & 62.91 \\
\hline
\end{tabular}

Table 4: Compressor Flow Limits

\begin{tabular}{|l|l|l|}
\hline S/NO. & Flow Limits & \\
\hline 1. & Surge Curve: & Inactive \\
\hline 2. & Speed & Flow \\
\hline 3. & Stone Wall Curve: & Inactive \\
\hline 4. & Field Flow Rate $\left(\mathrm{ACT} \mathrm{m}^{3} / \mathrm{h}\right)$ & 8330 \\
\hline 5. & Stone Wall Flow --- & \\
\hline 6. & Compressor Volume $\left(\mathrm{m}^{3}\right)$ & 10.00 \\
\hline 7. & Rotational inertia $\left(\mathrm{kg} / \mathrm{m}^{2}\right)$ & 6.000 \\
\hline 8. & Radius of gyration $(\mathrm{m})$ & 0.2000 \\
\hline 9. & Mass $(\mathrm{kg})$ & 150.0 \\
\hline 10. & Friction loss factor $(\mathrm{rad} / \mathrm{min})$ & 3.000 \\
\hline
\end{tabular}

Table 5: Compressor inlet Conditions

\begin{tabular}{|l|l|}
\hline & Overall \\
\hline Vapour/Phase Fraction & 1.0000 \\
\hline Temperature: $\left({ }^{0} \mathrm{C}\right)$ & 70.00 \\
\hline Pressure: $(\mathrm{kPa})$ & 208.0 \\
\hline Molar Flow $(\mathrm{kgmol} / \mathrm{h})$ & 607.7 \\
\hline Mass Flow $(\mathrm{kg} / \mathrm{h})$ & $1.759 \mathrm{e}+004$ \\
\hline Std Ideal LiqVol Flow $\left(\mathrm{m}^{3} / \mathrm{h}\right)$ & 20.00 \\
\hline Molar Enthalpy $(\mathrm{kJ} / \mathrm{kgmol})$ & 1283 \\
\hline Mass Enthalpy $(\mathrm{kJ} / \mathrm{kg})$ & 44.32 \\
\hline Molar Entropy $\left(\mathrm{kJ} / \mathrm{kgmol}^{0}{ }^{0} \mathrm{C}\right)$ & 116.2 \\
\hline Mass Entropy $\left(\mathrm{kJ} / \mathrm{kg}^{0} \mathrm{C}\right)$ & 4.015 \\
\hline
\end{tabular}




\begin{tabular}{|l|l|}
\hline Heat Flow $(\mathrm{kJ} / \mathrm{h})$ & $7.797 \mathrm{e}+005$ \\
\hline Molar Density $\left(\mathrm{kgmol} / \mathrm{m}^{3}\right)$ & $7.295 \mathrm{e}-002$ \\
\hline Mass Density $\left(\mathrm{kg} / \mathrm{m}^{3}\right)$ & 2.112 \\
\hline Std Ideal Liq Mass Density $\left(\mathrm{kg} / \mathrm{m}^{3}\right)$ & 879.6 \\
\hline Molar Heat Capacity $\left(\mathrm{kJ} / \mathrm{kgmol}^{0}{ }^{0} \mathrm{C}\right)$ & 29.00 \\
\hline Mass Heat Capacity $\left(\mathrm{kJ} / \mathrm{kg}^{0} \mathrm{C}\right)$ & 1.002 \\
\hline Thermal Conductivity $(\mathrm{W} / \mathrm{m}-\mathrm{K})$ & $2.775 \mathrm{e}-002$ \\
\hline Viscosity $(\mathrm{cP})$ & $2.093 \mathrm{e}-002$ \\
\hline Molecular Weight & 28.95 \\
\hline Z Factor & 0.9994 \\
\hline Cp/Cv & 1.406 \\
\hline Act. Vol. Flow $(\mathrm{m} 3 / \mathrm{h})$ & 8330 \\
\hline
\end{tabular}

Table 6: Compressor Outlet Conditions

\begin{tabular}{|l|l|}
\hline & Overall \\
\hline Vapour/Phase Fraction & 1.0000 \\
\hline Temperature: $\left({ }^{0} \mathrm{C}\right)$ & 172.8 \\
\hline Pressure: $(\mathrm{kPa})$ & 412.0 \\
\hline Molar Flow $(\mathrm{kgmol} / \mathrm{h})$ & 607.7 \\
\hline Mass Flow $(\mathrm{kg} / \mathrm{h})$ & $1.759 \mathrm{e}+004$ \\
\hline Std Ideal LiqVol Flow $\left(\mathrm{m}^{3} / \mathrm{h}\right)$ & 20.00 \\
\hline Molar Enthalpy $(\mathrm{kJ} / \mathrm{kgmol})$ & 4286 \\
\hline Mass Enthalpy $(\mathrm{kJ} / \mathrm{kg})$ & 148.0 \\
\hline Molar Entropy $\left(\mathrm{kJ} / \mathrm{kgmol}^{0}{ }^{0} \mathrm{C}\right)$ & 118.2 \\
\hline Mass Entropy $\left(\mathrm{kJ} / \mathrm{kg}^{-}{ }^{0} \mathrm{C}\right)$ & 4.083 \\
\hline Heat Flow $(\mathrm{kJ} / \mathrm{h})$ & $2.604 \mathrm{e}+006$ \\
\hline Molar Density $\left(\mathrm{kgmole} / \mathrm{m}^{3}\right)$ & 0.1111 \\
\hline Mass Density $\left(\mathrm{kg} / \mathrm{m}^{3}\right)$ & 3.215 \\
\hline Std Ideal Liq Mass Density $\left(\mathrm{kg} / \mathrm{m}^{3}\right)$ & 879.6 \\
\hline Molar Heat Capacity $\left(\mathrm{kJ} / \mathrm{kgmol}^{0}{ }^{0} \mathrm{C}\right)$ & 29.60 \\
\hline Mass Heat Capacity $\left(\mathrm{kJ} / \mathrm{kg}^{-}{ }^{0} \mathrm{C}\right)$ & 1.023 \\
\hline Thermal Conductivity $(\mathrm{W} / \mathrm{m}-\mathrm{K})$ & $3.421 \mathrm{e}-002$ \\
\hline Viscosity $(\mathrm{cP})$ & $2.524 \mathrm{e}-002$ \\
\hline Molecular Weight & 28.95 \\
\hline Z Factor & 1.000 \\
\hline Cp/Cv & 1.395 \\
\hline Act. Vol. Flow $\left(\mathrm{m}^{3} / \mathrm{h}\right)$ & 5471 \\
\hline
\end{tabular}

Table 7:Compressor Sizing Input Details

Unit operation type: Compressor

Equipment type: Gas Compressor - Centrifugal Horizontal

Compressor sizing input Details

\begin{tabular}{|l|l|}
\hline Operating capacity & $17591.48 \mathrm{~kg} / \mathrm{h}$ \\
\hline Operating adiabatic head & $7585.53 \mathrm{~m}$ \\
\hline Operating polytropic head & $7852.30 \mathrm{~m}$ \\
\hline Adiabatic efficiency & 71.7188 \\
\hline Polytropic efficiency & 74.2551 \\
\hline Operating gas power & $506.84 \mathrm{~kW}$ \\
\hline Capacity overdesign factor & 1,1000 \\
\hline Head overdesign factor & 1,1000 \\
\hline
\end{tabular}

Table 8: Compressor Sizing Data Output

Unit operation type: Compressor

Equipment type: Gas Compressor - Centrifugal Horizontal

Compressor sizing input Details

\begin{tabular}{|l|l|}
\hline Design capacity & $19350.63 \mathrm{~kg} / \mathrm{h}$ \\
\hline Design adiabatic head & $8344.1 \mathrm{~m}$ \\
\hline Design polytropic head & $8637.5 \mathrm{~m}$ \\
\hline Gas power & $613.16 \mathrm{~kW}$ \\
\hline Mechanical losses & $10.93 \mathrm{~kW}$ \\
\hline Design power & $624.09 \mathrm{~kW}$ \\
\hline Driver power & $900 \mathrm{hp}$ \\
\hline
\end{tabular}




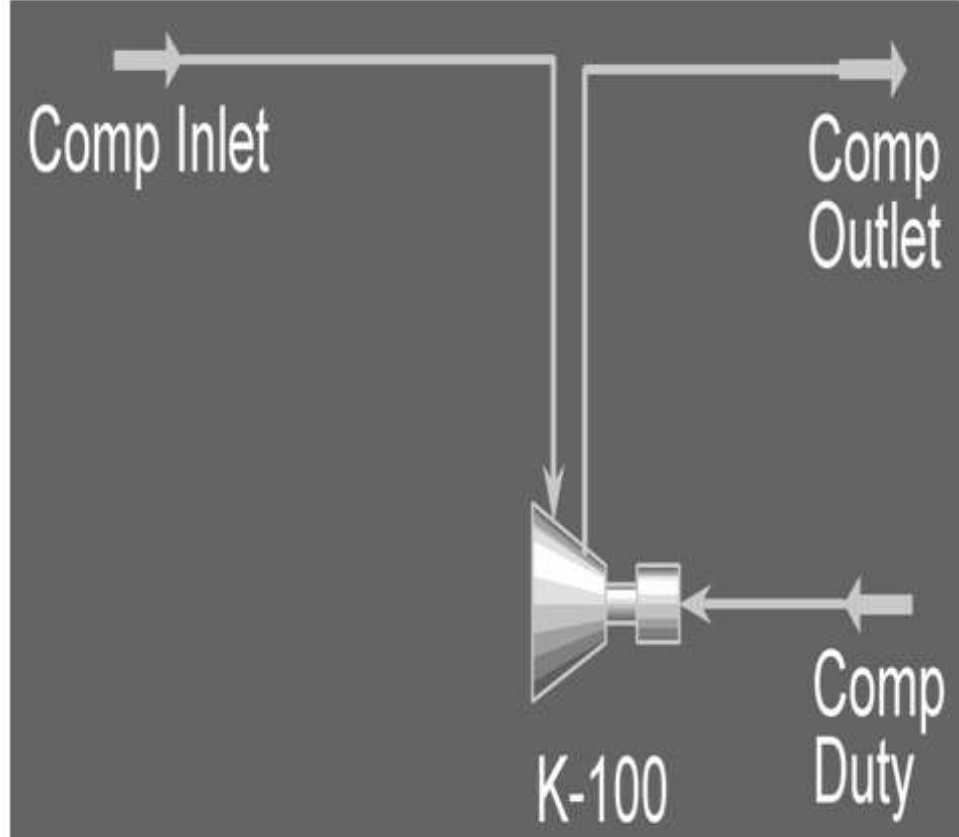

Figure .4: Compressor

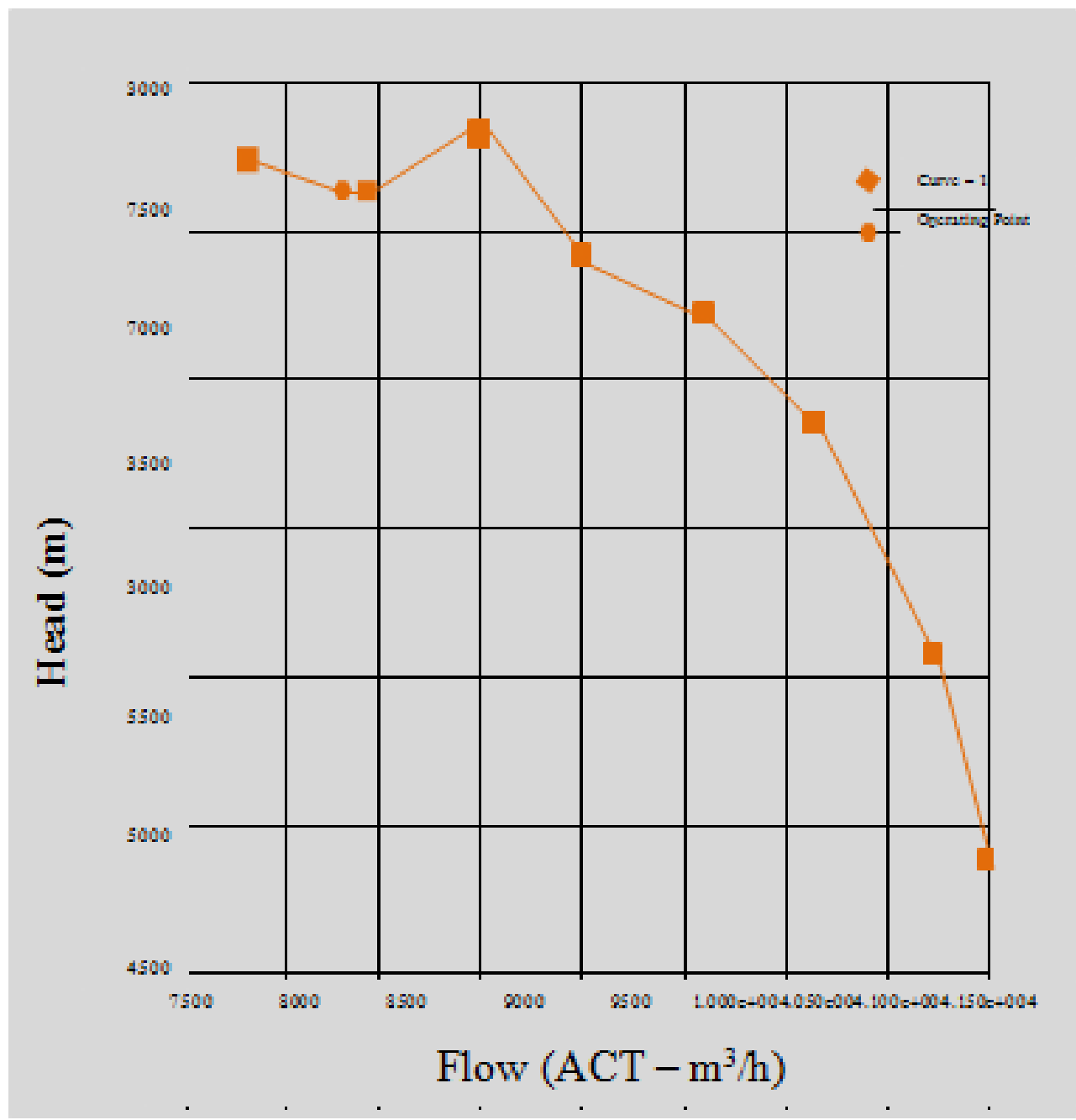

Figure 5: Head Curves of the Compressor 


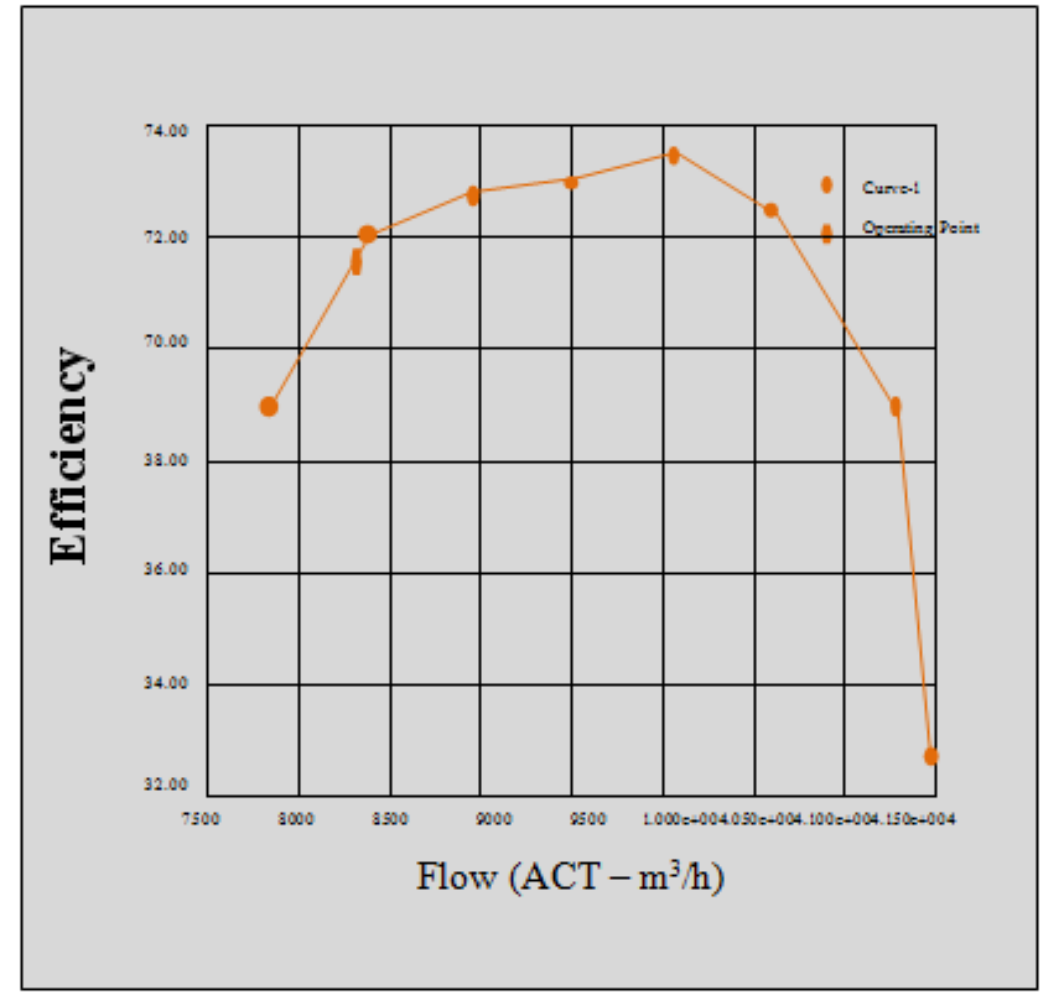

Fig. 6: Variation of Efficiency with Flow

\section{Simulated Results}

\section{Discussion Of Results}

From figure 6 (efficiency curves of the compressor) the efficiency increases when the flow $\left(\mathrm{m}^{3} / \mathrm{h}\right)$ is high and reduces when the level of the flow $\left(\mathrm{m}^{3} / \mathrm{h}\right)$ decreases.

From table 2 the adiabatic efficiency was found to be $71.7 \%$ while the polytrophic efficiency was $74.3 \%$.

Also from table 4 (compressor flow limits) the friction loss factor was found to be $3.00 \mathrm{rad} / \mathrm{min}$.

From the head curves of the compressor (figure 5), the head decreases when the flow $\left(\mathrm{m}^{3} / \mathrm{h}\right)$ increases.

The efficiency increases when the flow $\left(\mathrm{m}^{3} / \mathrm{h}\right)$ increases as shown in figure 6 .

\section{Conclusion}

This work set out to achieve the main goal of exploring ways for an effective energy saving which is expected to reduce energy cost, generate higher profit and increase capacity utilization. Energy loss which affects the output of the system was minimized. The empirical process heat loss, the actual values heat loss and the simulation model heat losss were found to be $2.56 \mathrm{Kw}, 9.81 \mathrm{~kW}$ and $10.93 \mathrm{~kW}$. The adiabatic efficiency and polytropic efficiency were found to be $71.7188 \%$ and $74.2551 \%$. Moreover, the mechanical losses was found to be $10.93 \mathrm{~kW}$.

\section{References}

[1]. Emshoff, J. R (2001)."Design and use of computer simulation Models". New York: Macmillan,

[2]. Chilton, C. H.(2002)"Cost Engineering in the Process Industries". New York: McGraw - Hill,

[3]. Engeda, Abraham (1999). "From the Crystal Palace to the pump room".Mechanical Engineering.ASME.

[4]. Elliott Company. "Past, Present, Future, 1910-2010"(PDF). Elliott. Retrieved 1 May 2011.

[5]. API ( 2002). Std 673-2002 Centrifugal Fans for Petroleum, Chemical and Gas Industry Services. New York: API

[6]. Whitfield, A. \& Baines, N. C. (1990).Design of Radial Turbomachinery.Longman Scientific and Technical.ISBN 0-470-21667-0.

[7]. Aungier, Ronald H. (2000). Centrifugal Compressors, A Strategy for Aerodynamic Design and Analysis. ASME Press. ISBN 07918-0093-8.

[8]. Saravanamuttoo, H.I.H., Rogers, G.F.C. and Cohen, H. (2001).Gas Turbine Theory.Prentice-Hall.ISBN 0-13-015847-X.

[9]. Baines, Nicholas C. (2005). Fundamentals of Turbocharging. Concepts ETI

[10]. . SAE/standards/power and propulsion/engines.SAE International.Retrieved 23 April 2011.

[11]. API ( 2002). Std 617-2002 Axial and Centrifugal Compressors and Expander-compressors for Petroleum, Chemical and Gas Industry Services. New York: API.

[12]. ASHRAE, American Society of Heating, Refrigeration and Air-Conditioning Engineers."Standards \& Guidelines"ASHRAE.Retrieved 23 April 2011.

[13]. API ( 2007). Std 672-2007 Packaged, Integrally Geared Centrifugal Air Compressors for Petroleum, Chemical, and Gas Industry Services. New York: API. 